\title{
POSITIVE PERIODIC SOLUTIONS FOR NONLINEAR DIFFERENCE EQUATIONS VIA A CONTINUATION THEOREM
}

\author{
GEN-QIANG WANG AND SUI SUN CHENG
}

Received 29 August 2003 and in revised form 4 February 2004

Based on a continuation theorem of Mawhin, positive periodic solutions are found for difference equations of the form $y_{n+1}=y_{n} \exp \left(f\left(n, y_{n}, y_{n-1}, \ldots, y_{n-k}\right)\right), n \in \mathbb{Z}$.

\section{Introduction}

There are several reasons for studying nonlinear difference equations of the form

$$
y_{n+1}=y_{n} \exp \left\{f\left(n, y_{n}, y_{n-1}, \ldots, y_{n-k}\right)\right\}, \quad n \in \mathbb{Z}=\{0, \pm 1, \pm 2, \ldots\} \text {, }
$$

where $f=f\left(t, u_{0}, u_{1}, \ldots, u_{k}\right)$ is a real continuous function defined on $\mathbb{R}^{k+2}$ such that

$$
f\left(t+\omega, u_{0}, \ldots, u_{k}\right)=f\left(t, u_{0}, \ldots, u_{k}\right), \quad\left(t, u_{0}, \ldots, u_{k}\right) \in \mathbb{R}^{k+2},
$$

and $\omega$ is a positive integer. For one reason, the well-known equations

$$
\begin{aligned}
& y_{n+1}=\lambda y_{n}, \\
& y_{n+1}=\mu y_{n}\left(1-y_{n}\right), \\
& y_{n+1}=y_{n} \exp \left\{\frac{\mu\left(1-y_{n}\right)}{K}\right\}, \quad K>0,
\end{aligned}
$$

are particular cases of (1.1). As another reason, (1.1) is intimately related to delay differential equations with piecewise constant independent arguments. To be more precise, let us recall that a solution of (1.1) is a real sequence of the form $\left\{y_{n}\right\}_{n \in \mathbb{Z}}$ which renders (1.1) into an identity after substitution. It is not difficult to see that solutions can be found when an appropriate function $f$ is given. However, one interesting question is whether there are any solutions which are positive and $\omega$-periodic, where a sequence $\left\{y_{n}\right\}_{n \in \mathbb{Z}}$ is said to be $\omega$-periodic if $y_{n+\omega}=y_{n}$, for $n \in \mathbb{Z}$. Positive $\omega$-periodic solutions of (1.1) are related to those of delay differential equations involving piecewise constant independent 
arguments:

$$
y^{\prime}(t)=y(t) f([t], y([t]), y([t-1]), y([t-2]), \ldots, y([t-k])), \quad t \in \mathbb{R},
$$

where $[x]$ is the greatest-integer function.

Such equations have been studied by several authors including Cooke and Wiener $[5,6]$, Shah and Wiener [9], Aftabizadeh et al. [1], Busenberg and Cooke [2], and so forth. Studies of such equations were motivated by the fact that they represent a hybrid of discrete and continuous dynamical systems and combine the properties of both differential and differential-difference equations. In particular, the following equation

$$
y^{\prime}(t)=a y(t)(1-y([t])),
$$

is in Carvalho and Cooke [3], where $a$ is constant.

By a solution of (1.4), we mean a function $y(t)$ which is defined on $\mathbb{R}$ and which satisfies the following conditions [1]: (i) $y(t)$ is continuous on $\mathbb{R}$; (ii) the derivative $y^{\prime}(t)$ exists at each point $t \in \mathbb{R}$ with the possible exception of the points $[t] \in \mathbb{R}$, where one-sided derivatives exist; and (iii) (1.4) is satisfied on each interval $[n, n+1) \subset \mathbb{R}$ with integral endpoints.

Theorem 1.1. Equation (1.1) has a positive w-periodic solution if and only if (1.4) has a positive $\omega$-periodic solution.

Proof. Let $y(t)$ be a positive $\omega$-periodic solution of (1.4). It is easy to see that for any $n \in \mathbb{Z}$

$$
y^{\prime}(t)=y(t) f(n, y(n), y(n-1), \ldots, y(n-k)), \quad n \leq t<n+1 .
$$

Integrating (1.6) from $n$ to $t$, we have

$$
y(t)=y(n) \exp ((t-n) f(n, y(n), y(n-1), \ldots, y(n-k))) .
$$

Since $\lim _{t \rightarrow(n+1)^{-}} y(t)=y(n+1)$, we see further that

$$
y(n+1)=y(n) \exp (f(n, y(n), y(n-1), \ldots, y(n-k))) .
$$

If we now let $y_{n}=y(n)$ for $n \in \mathbb{Z}$, then $\left\{y_{n}\right\}_{n \in \mathbb{Z}}$ is a positive $\omega$-periodic solution of (1.1).

Conversely, let $\left\{y_{n}\right\}_{n \in \mathbb{Z}}$ be a positive $\omega$-periodic solution of (1.1). Set $y(n)=y_{n}$, for $n \in \mathbb{Z}$, and let the function $y(t)$ on each interval $[n, n+1)$ be defined by $(1.7)$. Then it is not difficult to check that this function is a positive $\omega$-periodic solution of (1.4). The proof of Theorem 1.1 is complete.

Therefore, once the existence of a positive $\omega$-periodic solution of (1.1) can be demonstrated, we may then make immediate statements about the existence of positive $\omega$ periodic solutions of (1.4).

There appear to be several techniques (see, e.g., $[4,8,10]$ ) which can help to answer such a question. Among these techniques are fixed point theorems such as that of Krasnolselskii, Leggett-Williams, and others; and topological methods such as degree theories. 
Here we will invoke a continuation theorem of Mawhin for obtaining such solutions. More specifically, let $X$ and $Y$ be two Banach spaces and $L: \operatorname{Dom} L \subset X \rightarrow Y$ is a linear mapping and $N: X \rightarrow Y$ a continuous mapping [7, pages 39-40]. The mapping $L$ will be called a Fredholm mapping of index zero if $\operatorname{dim} \operatorname{Ker} L=\operatorname{codim} \operatorname{Im} L<+\infty$, and $\operatorname{Im} L$ is closed in $Y$. If $L$ is a Fredholm mapping of index zero, there exist continuous projectors $P: X \rightarrow X$ and $Q: Y \rightarrow Y$ such that $\operatorname{Im} P=\operatorname{Ker} L$ and $\operatorname{Im} L=\operatorname{Ker} Q=\operatorname{Im}(I-Q)$. It follows that $L_{\mid \operatorname{Dom} L \cap \operatorname{Ker} P}:(I-P) X \rightarrow \operatorname{Im} L$ has an inverse which will be denoted by $K_{P}$. If $\Omega$ is an open and bounded subset of $X$, the mapping $N$ will be called $L$-compact on $\bar{\Omega}$ if $Q N(\bar{\Omega})$ is bounded and $K_{P}(I-Q) N: \bar{\Omega} \rightarrow X$ is compact. Since $\operatorname{Im} Q$ is isomorphic to $\operatorname{Ker} L$ there exist an isomorphism $J: \operatorname{Im} Q \rightarrow \operatorname{Ker} L$.

Theorem 1.2 (Mawhin's continuation theorem). Let L be a Fredholm mapping of index zero, and let $N$ be L-compact on $\bar{\Omega}$. Suppose

(i) for each $\lambda \in(0,1), x \in \partial \Omega, L x \neq \lambda N x$;

(ii) for each $x \in \partial \Omega \cap \operatorname{Ker} L, Q N x \neq 0$ and $\operatorname{deg}(J Q N, \Omega \cap \operatorname{Ker}, 0) \neq 0$.

Then the equation $L x=N x$ has at least one solution in $\bar{\Omega} \cap \operatorname{dom} L$.

As a final remark in this section, note that if $\omega=1$, then a positive $\omega$-periodic solution of (1.1) is a constant sequence $\{c\}_{n \in \mathbb{Z}}$ that satisfies (1.1). Hence

$$
f(n, c, \ldots, c)=0, \quad n \in \mathbb{Z}
$$

Conversely, if $c>0$ such that $f(n, c, \ldots, c)=0$ for $n \in \mathbb{Z}$, then the constant sequence $\{c\}_{n \in \mathbb{Z}}$ is an $\omega$-periodic solution of (1.1). For this reason, we will assume in the rest of our discussion that $\omega$ is an integer greater than or equal to 2 .

\section{Existence criteria}

We will establish existence criteria based on combinations of the following conditions, where $D$ and $M$ are positive constants:
$\left(\mathrm{a}_{1}\right) f\left(t, e^{x_{0}}, \ldots, e^{x_{k}}\right)>0$ for $t \in \mathbb{R}$ and $x_{0}, \ldots, x_{k} \geq D$,
$\left(\mathrm{a}_{2}\right) f\left(t, e^{x_{0}}, \ldots, e^{x_{k}}\right)<0$ for $t \in \mathbb{R}$ and $x_{0}, \ldots, x_{k} \geq D$,
$\left(\mathrm{b}_{1}\right) f\left(t, e^{x_{0}}, \ldots, e^{x_{k}}\right)<0$ for $t \in \mathbb{R}$ and $x_{0}, \ldots, x_{k} \leq-D$,
$\left(\mathrm{b}_{2}\right) f\left(t, e^{x_{0}}, \ldots, e^{x_{k}}\right)>0$ for $t \in \mathbb{R}$ and $x_{0}, \ldots, x_{k} \leq-D$,
(c $\left.c_{1}\right) f\left(t, e^{x_{0}}, \ldots, e^{x_{k}}\right) \geq-M$ for $\left(t, e^{x_{0}}, \ldots, e^{x_{k}}\right) \in \mathbb{R}^{k+2}$,
( $\left.c_{2}\right) f\left(t, e^{x_{0}}, \ldots, e^{x_{k}}\right) \leq M$ for $\left(t, e^{x_{0}}, \ldots, e^{x_{k}}\right) \in \mathbb{R}^{k+2}$.

Theorem 2.1. Suppose either one of the following sets of conditions holds:

(i) $\left(\mathrm{a}_{1}\right),\left(\mathrm{b}_{1}\right)$, and $\left(\mathrm{c}_{1}\right)$, or,

(ii) $\left(a_{2}\right),\left(\mathrm{b}_{2}\right)$, and $\left(\mathrm{c}_{1}\right)$, or,

(iii) $\left(\mathrm{a}_{1}\right),\left(\mathrm{b}_{1}\right)$, and $\left(\mathrm{c}_{2}\right)$, or

(iv) $\left(\mathrm{a}_{2}\right),\left(\mathrm{b}_{2}\right)$, and $\left(\mathrm{c}_{2}\right)$.

Then (1.1) has a positive w-periodic solution.

We only give the proof in case $\left(a_{1}\right),\left(b_{1}\right)$, and $\left(c_{1}\right)$ hold, since the other cases can be treated in similar manners. 
We first need some basic tools. First of all, for any real sequence $\left\{u_{n}\right\}_{n \in \mathbb{Z}}$, we define a nonstandard "summation" operation

$$
\bigoplus_{n=\alpha}^{\beta} u_{n}= \begin{cases}\sum_{n=\alpha}^{\beta} u_{n}, & \alpha \leq \beta, \\ 0, & \beta=\alpha-1, \\ -\sum_{n=\beta+1}^{\alpha-1} u_{n}, & \beta<\alpha-1 .\end{cases}
$$

It is then easy to see if $\left\{x_{n}\right\}_{n \in \mathbb{Z}}$ is a $\omega$-periodic solution of the following equation

$$
x_{n}=x_{0}+\bigoplus_{i=0}^{n-1} f\left(i, e^{x_{i}}, e^{x_{i-1}}, \ldots, e^{x_{i-k}}\right), \quad n \in \mathbb{Z},
$$

then $\left\{y_{n}\right\}_{n \in \mathbb{Z}}=\left\{e^{x_{n}}\right\}_{n \in \mathbb{Z}}$ is a positive $\omega$-periodic solution of (1.1). We will therefore seek an $\omega$-periodic solution of (2.2).

Let $X_{\omega}$ be the Banach space of all real $\omega$-periodic sequences of the form $x=\left\{x_{n}\right\}_{n \in \mathbb{Z}}$, and endowed with the usual linear structure as well as the norm $\|x\|_{1}=\max _{0 \leq i \leq \omega-1}\left|x_{i}\right|$. Let $Y_{\omega}$ be the Banach space of all real sequences of the form $y=\left\{y_{n}\right\}_{n \in \mathbb{Z}}=\left\{n \alpha+h_{n}\right\}_{n \in \mathbb{Z}}$ such that $y_{0}=0$, where $\alpha \in \mathbb{R}$ and $\left\{h_{n}\right\}_{n \in \mathbb{Z}} \in X_{\omega}$, and endowed with the usual linear structure as well as the norm $\|y\|_{2}=|\alpha|+\|h\|_{1}$. Let the zero element of $X_{\omega}$ and $Y_{\omega}$ be denoted by $\theta_{1}$ and $\theta_{2}$ respectively.

Define the mappings $L: X_{\omega} \rightarrow Y_{\omega}$ and $N: X_{\omega} \rightarrow Y_{\omega}$, respectively, by

$$
\begin{gathered}
(L x)_{n}=x_{n}-x_{0}, \quad n \in \mathbb{Z}, \\
(N x)_{n}=\bigoplus_{i=0}^{n-1} f\left(i, e^{x_{i}}, e^{x_{i-1}}, \ldots, e^{x_{i-k}}\right), \quad n \in \mathbb{Z} .
\end{gathered}
$$

Let

$$
\bar{h}_{n}=\bigoplus_{i=0}^{n-1} f\left(i, e^{x_{i}}, e^{x_{i-1}}, \ldots, e^{x_{i-k}}\right)-\frac{n}{\omega} \bigoplus_{i=0}^{\omega-1} f\left(i, e^{x_{i}}, e^{x_{i-1}}, \ldots, e^{x_{i-k}}\right), \quad n \in \mathbb{Z} .
$$

Since $\bar{h}=\left\{\bar{h}_{n}\right\}_{n \in \mathbb{Z}} \in X_{\omega}$ and $\bar{h}_{0}=0, N$ is a well-defined operator from $X_{\omega}$ to $Y_{\omega}$. On the other hand, direct calculation leads to $\operatorname{Ker} L=\left\{x \in X_{\omega} \mid x_{n}=x_{0}, n \in \mathbb{Z}, x_{0} \in \mathbb{R}\right\}$ and $\operatorname{Im} L=X_{\omega} \cap Y_{\omega}$. Let us define $P: X_{\omega} \rightarrow X_{\omega}$ and $Q: Y_{\omega} \rightarrow Y_{\omega}$, respectively, by

$$
\begin{gathered}
(P x)_{n}=x_{0}, \quad n \in \mathbb{Z}, \text { for } x=\left\{x_{n}\right\}_{n \in \mathbb{Z}} \in X_{\omega}, \\
(Q y)_{n}=n \alpha \quad \text { for } y=\left\{n \alpha+h_{n}\right\}_{n \in \mathbb{Z}} \in Y_{\omega} .
\end{gathered}
$$

The operators $P$ and $Q$ are projections and $X_{\omega}=\operatorname{Ker} P \oplus \operatorname{Ker} L, Y_{\omega}=\operatorname{Im} L \oplus \operatorname{Im} Q$. It is easy to see that $\operatorname{dim} \operatorname{Ker} L=1=\operatorname{dim} \operatorname{Im} Q=\operatorname{codim} \operatorname{Im} L$, and that

$$
\operatorname{Im} L=\left\{y \in X_{\omega} \mid y_{0}=0\right\} \subset Y_{\omega} .
$$

It follows that $\operatorname{Im} L$ is closed in $Y_{\omega}$. Thus the following lemma is true. 
Lemma 2.2. The mapping $L$ defined by (2.3) $L$ is a Fredholm mapping of index zero.

Next we recall that a subset $S$ of a Banach space $X$ is relatively compact if, and only if, for each $\varepsilon>0$, it has a finite $\varepsilon$-net.

LeMma 2.3. A subset $S$ of $X_{\omega}$ is relatively compact if and only if $S$ is bounded.

Proof. It is easy to see that if $S$ is relatively compact in $X_{\omega}$, then $S$ is bounded. Conversely, if the subset $S$ of $X_{\omega}$ is bounded, then there is a subset

$$
\Gamma:=\left\{x \in X_{\omega} \mid\|x\|_{1} \leq H\right\}
$$

where $H$ is a positive constant, such that $S \subset \Gamma$. It suffices to show that $\Gamma$ is relatively compact in $X_{\omega}$. Note that for each $\varepsilon>0$, we may choose numbers $y_{0}<y_{1}<\cdots<y_{l}$ such that $y_{0}=-H, y_{l}=H$ and $y_{i+1}-y_{i}<\varepsilon$ for $i=0, \ldots, l-1$. Then

$$
\left\{v=\left\{v_{n}\right\}_{n \in \mathbb{Z}} \in X_{\omega} \mid v_{j} \in\left\{y_{0}, y_{1}, \ldots, y_{l-1}\right\}, j=0, \ldots, \omega-1\right\}
$$

is a finite $\varepsilon$-net of $\Gamma$. This completes the proof.

Lemma 2.4. Let $L$ and $N$ be defined by (2.3) and (2.4), respectively. Suppose $\Omega$ is an open bounded subset of $X_{\omega}$. Then $N$ is L-compact on $\bar{\Omega}$.

Proof. From (2.4), (2.5), and (2.7), we see that for any $x=\left\{x_{n}\right\}_{n \in \mathbb{Z}} \in \bar{\Omega}$,

$$
(Q N x)_{n}=\frac{n}{\omega} \bigoplus_{i=0}^{\omega-1} f\left(i, e^{x_{i}}, e^{x_{i-1}}, \ldots, e^{x_{i-k}}\right), \quad n \in \mathbb{Z}
$$

Thus

$$
\|Q N x\|_{2}=\left\|\frac{n}{\omega} \bigoplus_{i=0}^{\omega-1} f\left(i, e^{x_{i}}, e^{x_{i-1}}, \ldots, e^{x_{i-k}}\right)\right\|_{2}=\frac{1}{\omega}\left|\sum_{i=0}^{\omega-1} f\left(i, e^{x_{i}}, e^{x_{i-1}}, \ldots, e^{x_{i-k}}\right)\right|
$$

so that $Q N(\bar{\Omega})$ is bounded. We denote the inverse of the mapping $\left.L\right|_{\operatorname{Dom} L \cap \operatorname{Ker} P}:(I-$ $P) X \rightarrow \operatorname{Im} L$ by $K_{P}$. Direct calculations lead to

$$
\left(K_{P}(I-Q) N x\right)_{n}=\bigoplus_{i=0}^{n-1} f\left(i, e^{x_{i}}, e^{x_{i-1}}, \ldots, e^{x_{i-k}}\right)-\frac{n}{\omega} \bigoplus_{i=0}^{\omega-1} f\left(i, e^{x_{i}}, e^{x_{i-1}}, \ldots, e^{x_{i-k}}\right) .
$$

It is easy to see that

$$
\left\|K_{P}(I-Q) N x\right\|_{1} \leq 2\left|\bigoplus_{i=0}^{\omega-1} f\left(i, e^{x_{i}}, e^{x_{i-1}}, \ldots, e^{x_{i-k}}\right)\right|
$$


Noting that $\bar{\Omega}$ is a closed and bounded subset of $X_{\omega}$ and $f$ is continuous on $\mathbb{R}^{k+2}$, relation (2.14) implies that $K_{P}(I-Q) N(\bar{\Omega})$ is bounded in $X_{\omega}$. In view of Lemma 2.3, $K_{P}(I-$ Q) $N(\bar{\Omega})$ is relatively compact in $X_{\omega}$. Since the closure of a relatively compact set is relatively compact, $\overline{K_{P}(I-Q) N(\bar{\Omega})}$ is relatively compact in $X_{\omega}$ and hence $N$ is $L$-compact on $\bar{\Omega}$. This completes the proof.

Now, we consider the following equation

$$
x_{n}-x_{0}=\lambda \bigoplus_{i=0}^{n-1} f\left(i, e^{x_{i}}, e^{x_{i-1}}, \ldots, e^{x_{i-k}}\right), \quad n \in \mathbb{Z},
$$

where $\lambda \in(0,1)$.

Lemma 2.5. Suppose $\left(\mathrm{a}_{1}\right),\left(\mathrm{b}_{1}\right)$, and $\left(\mathrm{c}_{1}\right)$ are satisfied. Then for any $\omega$-periodic solution $x=\left\{x_{n}\right\}_{n \in \mathbb{Z}}$ of (2.15),

$$
\|x\|_{1}=\max _{0 \leq i \leq \omega-1}\left|x_{i}\right| \leq D+4 \omega M .
$$

Proof. Let $x=\left\{x_{n}\right\}_{n \in \mathbb{Z}}$ be a $\omega$-periodic solution $x=\left\{x_{n}\right\}_{n \in \mathbb{Z}}$ of (2.15). Then

$$
\bigoplus_{i=0}^{\omega-1} f\left(i, e^{x_{i}}, e^{x_{i-1}}, \ldots, e^{x_{i-k}}\right)=0
$$

If we write

$$
\begin{aligned}
& G_{n}^{+}=\max \left\{f\left(n, e^{x_{n}}, e^{x_{n-1}}, \ldots, e^{x_{n-k}}\right), 0\right\}, \quad n \in \mathbb{Z}, \\
& G_{n}^{-}=\max \left\{-f\left(n, e^{x_{n}}, e^{x_{n-1}}, \ldots, e^{x_{n-k}}\right), 0\right\}, \quad n \in \mathbb{Z},
\end{aligned}
$$

then $\left\{G_{n}^{+}\right\}_{n \in \mathbb{Z}}$ and $\left\{G_{n}^{-}\right\}_{n \in \mathbb{Z}}$ are nonnegative real sequences and

$$
f\left(n, e^{x_{n}}, e^{x_{n-1}}, \ldots, e^{x_{n-k}}\right)=G_{n}^{+}-G_{n}^{-}, \quad n \in \mathbb{Z},
$$

as well as

$$
\left|f\left(n, e^{x_{n}}, e^{x_{n-1}}, \ldots, e^{x_{n-k}}\right)\right|=G_{n}^{+}+G_{n}^{-}, \quad n \in \mathbb{Z} .
$$

In view of $\left(c_{1}\right)$ and (2.19), we have

$$
\left|G_{n}^{-}\right|=G_{n}^{-} \leq M, \quad n \in \mathbb{Z}
$$

Thus

$$
\bigoplus_{i=0}^{\omega-1} G_{i}^{-} \leq \omega M
$$

and in view of (2.17), (2.20), and (2.23),

$$
\bigoplus_{i=0}^{\omega-1} G_{i}^{+}=\bigoplus_{i=0}^{\omega-1} G_{i}^{-} \leq \omega M
$$


By (2.21) and (2.24), we know that

$$
\bigoplus_{i=0}^{\omega-1}\left|f\left(i, e^{x_{i}}, e^{x_{i-1}}, \ldots, e^{x_{i-k}}\right)\right| \leq 2 \omega M
$$

Let $x_{\alpha}=\max _{0 \leq i \leq \omega-1} x_{i}$ and $x_{\beta}=\min _{0 \leq i \leq \omega-1} x_{i}$, where $0 \leq \alpha, \beta \leq \omega-1$. By (2.15), we have

$$
\begin{aligned}
x_{\alpha}-x_{\beta} & =\left|x_{\alpha}-x_{\beta}\right|=\lambda\left|\bigoplus_{i=0}^{\alpha-1} f\left(i, e^{x_{i}}, e^{x_{i-1}}, \ldots, e^{x_{i-k}}\right)-\bigoplus_{i=0}^{\beta-1} f\left(i, e^{x_{i}}, e^{x_{i-1}}, \ldots, e^{x_{i-k}}\right)\right| \\
& \leq 2 \bigoplus_{i=0}^{\omega-1}\left|f\left(i, e^{x_{i}}, e^{x_{i-1}}, \ldots, e^{x_{i-k}}\right)\right| \leq 4 \omega M .
\end{aligned}
$$

If there is some $x_{l}, 0 \leq l \leq \omega-1$, such that $\left|x_{l}\right|<D$, then in view of (2.15) and (2.25), for any $n \in\{0,1, \ldots, \omega-1\}$, we have

$$
\begin{aligned}
\left|x_{n}\right| & =\left|x_{l}\right|+\left|x_{n}-x_{l}\right| \\
& \leq D+\left|\bigoplus_{i=0}^{n-1} f\left(i, e^{x_{i}}, e^{x_{i-1}}, \ldots, e^{x_{i-k}}\right)-\bigoplus_{i=0}^{l-1} f\left(i, e^{x_{i}}, e^{x_{i-1}}, \ldots, e^{x_{i-k}}\right)\right| \\
& \leq D+2 \bigoplus_{i=0}^{\omega-1}\left|f\left(i, e^{x_{i}}, e^{x_{i-1}}, \ldots, e^{x_{i-k}}\right)\right| \\
& \leq D+4 \omega M .
\end{aligned}
$$

Otherwise, by $\left(\mathrm{a}_{1}\right),\left(\mathrm{b}_{1}\right)$, and (2.17), $x_{\alpha} \geqslant D$ and $x_{\beta} \leq-D$. From (2.26), we have

$$
\begin{aligned}
& x_{\alpha} \leq x_{\beta}+4 \omega M \leq-D+4 \omega M, \\
& x_{\beta} \geq x_{\alpha}-4 \omega M \geq D-4 \omega M .
\end{aligned}
$$

It follows that

$$
D-4 \omega M \leq x_{\beta} \leq x_{n} \leq x_{\alpha} \leq-D+4 \omega M, \quad 0 \leq n \leq \omega-1,
$$

or

$$
\left|x_{n}\right| \leq D+4 \omega M, \quad 0 \leq n \leq \omega-1 .
$$

This completes the proof.

We now turn to the proof of Theorem 2.1. Let $L, N, P$ and $Q$ be defined by (2.3), (2.4), (2.6), and (2.7), respectively. Set

$$
\Omega=\left\{x \in X_{\omega} \mid\|x\|_{1}<\bar{D}\right\},
$$

where $\bar{D}$ is a fixed number which satisfies $\bar{D}>D+4 \omega M$. It is easy to see that $\Omega$ is an open and bounded subset of $X_{\omega}$. Furthermore, in view of Lemma 2.2 and Lemma 2.4, $L$ is a Fredholm mapping of index zero and $N$ is $L$-compact on $\bar{\Omega}$. Noting that $\bar{D}>D+4 \omega M$, 
by Lemma 2.5, for each $\lambda \in(0,1)$ and $x \in \partial \Omega, L x \neq \lambda N x$. Next, note that a sequence $x=\left\{x_{n}\right\}_{n \in \mathbb{Z}} \in \partial \Omega \cap \operatorname{Ker} L$ must be constant: $\left\{x_{n}\right\}_{n \in \mathbb{Z}}=\{\bar{D}\}_{n \in \mathbb{Z}}$ or $\left\{x_{n}\right\}_{n \in \mathbb{Z}}=\{-\bar{D}\}_{n \in \mathbb{Z}}$. Hence by $\left(\mathrm{a}_{1}\right),\left(\mathrm{b}_{1}\right)$, and (2.11),

$$
(Q N x)_{n}=\frac{n}{\omega} \bigoplus_{i=0}^{\omega-1} f\left(i, e^{x_{0}}, \ldots, e^{x_{0}}\right), \quad n \in \mathbb{Z},
$$

so

$$
Q N x \neq \theta_{2} \text {. }
$$

The isomorphism $J: \operatorname{Im} Q \rightarrow \operatorname{Ker} L$ is defined by $(J(n \alpha))_{n}=\alpha$, for $\alpha \in \mathbb{R}, n \in \mathbb{Z}$. Then

$$
(J Q N x)_{n}=\frac{1}{\omega} \bigoplus_{i=0}^{\omega-1} f\left(i, e^{x_{0}}, \ldots, e^{x_{0}}\right) \neq 0, \quad n \in \mathbb{Z} .
$$

In particular, we see that if $\left\{x_{n}\right\}_{n \in \mathbb{Z}}=\{\bar{D}\}_{n \in \mathbb{Z}}$, then

$$
(J Q N x)_{n}=\frac{1}{\omega} \bigoplus_{i=0}^{\omega-1} f\left(i, e^{\bar{D}}, \ldots, e^{\bar{D}}\right)>0, \quad n \in \mathbb{Z},
$$

and if $\left\{x_{n}\right\}_{n \in \mathbb{Z}}=\{-\bar{D}\}_{n \in \mathbb{Z}}$, then

$$
(J Q N x)_{n}=\frac{1}{\omega} \bigoplus_{i=0}^{\omega-1} f\left(i, e^{-\bar{D}}, \ldots, e^{-\bar{D}}\right)<0, \quad n \in \mathbb{Z} .
$$

Consider the mapping

$$
H(x, s)=s x+(1-s) J Q N x, \quad 0 \leq s \leq 1 .
$$

From (2.35) and (2.37), for each $s \in[0,1]$ and $\left\{x_{n}\right\}_{n \in \mathbb{Z}}=\{\bar{D}\}_{n \in \mathbb{Z}}$, we have

$$
(H(x, s))_{n}=s \bar{D}+(1-s) \frac{1}{\omega} \bigoplus_{i=0}^{\omega-1} f\left(i, e^{\bar{D}}, \ldots, e^{\bar{D}}\right)>0, \quad n \in \mathbb{Z} .
$$

Similarly, from (2.36) and (2.37), for each $s \in[0,1]$ and $\left\{x_{n}\right\}_{n \in \mathbb{Z}}=\{-\bar{D}\}_{n \in \mathbb{Z}}$, we have

$$
(H(x, s))_{n}=-s \bar{D}+(1-s) \frac{1}{\omega} \bigoplus_{i=0}^{\omega-1} f\left(i, e^{-\bar{D}}, \ldots, e^{-\bar{D}}\right)<0, \quad n \in \mathbb{Z} .
$$

By (2.38) and (2.39), $H(x, s)$ is a homotopy. This shows that

$$
\operatorname{deg}\left(J Q N x, \Omega \cap \operatorname{Ker} L, \theta_{1}\right)=\operatorname{deg}\left(-x, \Omega \cap \operatorname{Ker} L, \theta_{1}\right) \neq 0 .
$$

By Theorem 1.2, we see that equation $L x=N x$ has at least one solution in $\bar{\Omega} \cap \operatorname{Dom} L$. In other words, (2.2) has an $\omega$-periodic solution $x=\left\{x_{n}\right\}_{n \in \mathbb{Z}}$, and hence $\left\{e^{x_{n}}\right\}_{n \in \mathbb{Z}}$ is a positive $\omega$-periodic solution of (1.1).

COROLlary 2.6. Under the same assumption of Theorem 1.1, (1.4) has a positive w-periodic solution. 


\section{Examples}

Consider the difference equation

$$
y_{n+1}=y_{n} \exp \left(r(n)\left(\frac{a(n)-y_{n-k}}{a(n)+c(n) r(n) y_{n-k}}\right)^{\delta}\right), \quad n \in \mathbb{Z},
$$

and the semi-discrete "food-limited" population model of

$$
y^{\prime}(t)=y(t) r([t])\left(\frac{a([t])-y([t-k])}{a([t])+c([t]) r([t]) y([t-k])}\right)^{\delta}, \quad t \in \mathbb{R}
$$

In (3.1) or (3.2), $r, a$, and $c$ belong to $C(\mathbb{R},(0, \infty))$, and $r(t+\omega)=r(t), a(t+\omega)=a(t)$, $c(t+\omega)=c(t)$ and $\delta$ is a positive odd integer. Letting

$$
\begin{gathered}
M=\max _{0 \leq t \leq \omega} r(t), \\
f\left(t, u_{0}, u_{1}, \ldots, u_{k}\right)=r(t)\left(\frac{a(t)-u_{k}}{a(t)+c(t) r(t) u_{k}}\right)^{\delta}, \\
D=\max _{0 \leq t \leq \omega}|\ln a(t)|+\varepsilon_{0}, \quad \varepsilon_{0}>0 .
\end{gathered}
$$

It is easy to verify that the conditions $\left(\mathrm{a}_{2}\right),\left(\mathrm{b}_{2}\right)$, and $\left(\mathrm{c}_{1}\right)$ are satisfied. By Theorem 2.1 and Corollary 2.6, we know that (3.1) and (3.2) have positive $\omega$-periodic solutions.

As another example, consider the semi-discrete Michaelis-Menton model

$$
y^{\prime}(t)=y(t) r([t])\left(1-\sum_{i=0}^{k} \frac{a_{i}([t]) y([t-i])}{1+c_{i}([t]) y([t-i])}\right), \quad t \in \mathbb{R}
$$

and its associated difference equation

$$
y_{n+1}=y_{n} \exp \left(r(n)\left(1-\sum_{i=0}^{k} \frac{a_{i}(n) y_{n-i}}{1+c_{i}(n) y_{n-i}}\right)\right), \quad n \in \mathbb{Z} .
$$

In (3.4) and (3.5), $r, a_{i}$, and $c_{i}$ belong to $C(\mathbb{R},(0, \infty)), r(t+\omega)=r(t), a_{i}(t+\omega)=a_{i}(t)$ and $c_{i}(t+\omega)=c_{i}(t)$ for $i=0,1, \ldots, k$ and $t \in \mathbb{R}$, and $\sum_{i=0}^{k} a_{i}(t) / c_{i}(t)>1$. Letting

$$
f\left(t, u_{0}, u_{1}, \ldots, u_{k}\right)=r(t)\left(1-\sum_{i=0}^{k} \frac{a_{i}(t) u_{i}}{1+c_{i}(t) u_{i}}\right)
$$

then

$$
f\left(t, e^{x_{0}}, e^{x_{1}}, \ldots, e^{x_{k}}\right)=r(t)\left(1-\sum_{i=0}^{k} \frac{a_{i}(t) e^{x_{i}}}{1+c_{i}(t) e^{x_{i}}}\right)
$$


Since

$$
\begin{gathered}
\lim _{x_{0}, \ldots, x_{k} \rightarrow+\infty} \min _{0 \leq t \leq \omega} \sum_{i=0}^{k} \frac{a_{i}(t) e^{x_{i}}}{1+c_{i}(t) e^{x_{i}}}>1, \\
\lim _{x_{0}, \ldots, x_{k} \rightarrow-\infty} \max _{0 \leq t \leq \omega} \sum_{i=0}^{k} \frac{a_{i}(t) e^{x_{i}}}{1+c_{i}(t) e^{x_{i}}}=0,
\end{gathered}
$$

we can choose $M=\max _{0 \leq t \leq \omega} r(t)$ and some positive number $D$ such that conditions $\left(\mathrm{a}_{2}\right)$, $\left(b_{2}\right)$, and $\left(c_{1}\right)$ are satisfied. By Theorem 2.1 and Corollary 2.6, (3.4), and (3.5) have positive $\omega$-periodic solution.

\section{References}

[1] A. R. Aftabizadeh, J. Wiener, and J.-M. Xu, Oscillatory and periodic solutions of delay differential equations with piecewise constant argument, Proc. Amer. Math. Soc. 99 (1987), no. 4, 673679.

[2] S. Busenberg and K. Cooke, Vertically Transmitted Diseases, Biomathematics, vol. 23, SpringerVerlag, Berlin, 1993.

[3] L. A. V. Carvalho and K. L. Cooke, A nonlinear equation with piecewise continuous argument, Differential Integral Equations 1 (1988), no. 3, 359-367.

[4] S. Cheng and G. Zhang, Positive periodic solutions of a discrete population model, Funct. Differ. Equ. 7 (2000), no. 3-4, 223-230.

[5] K. L. Cooke and J. Wiener, Retarded differential equations with piecewise constant delays, J. Math. Anal. Appl. 99 (1984), no. 1, 265-297.

[6] _ A survey of differential equations with piecewise continuous arguments, Delay Differential Equations and Dynamical Systems (Claremont, Calif, 1990), Lecture Notes in Mathematics, vol. 1475, Springer, Berlin, 1991, pp. 1-15.

[7] R. E. Gaines and J. L. Mawhin, Coincidence Degree, and Nonlinear Differential Equations, Lecture Notes in Mathematics, vol. 568, Springer-Verlag, Berlin, 1977.

[8] M. I. Gil' and S. S. Cheng, Periodic solutions of a perturbed difference equation, Appl. Anal. 76 (2000), no. 3-4, 241-248.

[9] S. M. Shah and J. Wiener, Advanced differential equations with piecewise constant argument deviations, Int. J. Math. Math. Sci. 6 (1983), no. 4, 671-703.

[10] G. Zhang and S. S. Cheng, Positive periodic solutions for discrete population models, Nonlinear Funct. Anal. Appl. 8 (2003), no. 3, 335-344.

Gen-Qiang Wang: Department of Computer Science, Guangdong Polytechnic Normal University, Guangzhou, Guangdong 510665, China

E-mail address:w7633@hotmail.com

Sui Sun Cheng: Department of Mathematics, Tsing Hua University, Hsinchu, Taiwan 30043, China E-mail address: sscheng@math.nthu.edu.tw 